\title{
LA VALLÉE DE JOUX
}

RENE MEYLAN

\section{BUT DE L'EXCURSION}

Le but de l'excursion est d'introduire dans une région qui est la plus variée et la plus intéressante de tout le Jura plissé. Si le haut Jura peut être considéré comme le plus beau système plissé qui soit, le Jura vaudois en est un élément essentiel où la Vallée de Joux apporte son originalité de bassin fermé.

\section{RENSEIGNEMENTS TECHNIQUES}

Voies d'accès - hôtels

A. Par la ligne Lausanne-Vallorbe. Au Day, embranchement pour le Pont. Par route, de Vallorbe $(77 \circ \mathrm{m})$ au Pont $(1008 \mathrm{~m}): 8 \mathrm{~km}$. Par route, de Croy-Romainmôtier $(645 \mathrm{~m})$ par le col de Petra Felix $(1144 \mathrm{~m})$, au Pont $(1008 \mathrm{~m}): 16 \mathrm{~km}$. Par route, de Cossonay (57o m), par le col du Molendruz (1180 m), au Pont (1008 m): $18 \mathrm{~km}$.

B. Par voie ferrée, de Morges à l'Isle $(667 \mathrm{~m})$ et de l'Isle, par le Molendruz, au Pont: $10 \mathrm{~km}$. Par voie ferrée, de Morges à Bière $(705 \mathrm{~m})$, par le col du Marchairuz $(1+47 \mathrm{~m})$, au Brassus $(1036 \mathrm{~m}): 4 \mathrm{~h}$.

C. Par voie ferrée, de Nyon à La Cure $(1232 \mathrm{~m}$ ) et par route de La Cure au Brassus (1036 m) : $17 \mathrm{~km}$.

D. Par route, de Mouthe $(938 \mathrm{~m})$, par le Risoud, au Pont: $15 \mathrm{~km}$.

Les relations internes sont assurées par la voie ferrée du Pont au Brassus ( 8 courses par jour dans chaque sens) et par un service automobile entre Le Pont et Le Sentier, par la rive orientale du lac.

La plupart des localités sont dotées d'hôtels. Le Brassus: Hôtel de France (35 lits)-Hôtel de la Lande (2o lits) - Le Sentier: Hôtel de Ville (14 lits) - Hôtel du Lion d'Or (25 lits) Hôtel de l'Union (1o lits) - Hôtel Bellevue, au Rocheray, (25 lits) - Les Bioux: Hôtel des Trois-Suisses (15 lits). L'Abbaye: Hôtel de Ville (12 lits). - Le Pont: Hôtel de la Truite (16 lits). - Auberge de Jeunesse - Les Charbonnières: Hôtel du Cygne (1o lits).

Le col du Marchairuz et le col du Molendruz ont de bonnes auberges, dites Asiles. Plusieurs chalets ont un service de restauration pendant la saison d'été (Dent de Vaulion, Châtel, Mont d'Orzeires).

\section{CARTES - BIBLIOGRAPHIE}

Cartes

Carte nationale de la Suisse au 1:25 ooo: 1221 Le Sentier, 1222 Cossonay, 1206 Orbe, 1201 Mouthe, 1241 Marchairuz.

Carte spéciale du Jura, Feuille 6, au 1:50 o00, Kümmerly \& Frey, Berne.

Carte nationale de la Suisse au 1:50000: 1250 Vallée de Joux, 1251 La Sarraz.

\section{Cartes géologiques}

D. Aubert: Atlas géologique de la Suisse, F.17 (Vallée de Joux), 1:25 ooo. A. B. Tutein Nolthenius: Carte des environs de Vallorbe, Comm. géol. Soc. Helv. Sc. nat. carte spec. N. 92, 1:25 ooo. W. Custer et D. Aubert: Atlas géol. de la Suisse, F. 5, 1:25 000.

\section{Bibliographie}

D. Aubert: Monographie géol. de la Vallée de Joux. (Mat. pour la carte géol. de la Suisse, 1943. S. Aubert: La flore de la Vallée de Joux (Bull. Soc. vaud. Sc. nat., vol. 36). H. Gutersohn: Jura, Berne, 1958. H. Gutersohn: Landschaften der Schweiz, Zurich, 195o. R. Meylan: La Vallée de Joux; étude de géographie humaine (Bull. Soc. neuch. de géogr., T38).

\section{ITINERAIRE}

Vallorbe-Résurgence de l'Orbe-Grottes aux Fées-Col de la Pierre-Punex-Entonnoirs du lac Brenet-Les Charbonnières-Les Epinettes-Le Pont-Le Mont du Lac-Source de la LyonneL'Abbaye-Le Pont-Les Charbonnières-Le Séchey-Le Lieu-Combenoire-La Golisse-Le Sentier-Chez le Maître-Les Piguet-dessous-Chez Tribillet-Pré Rodet-Le Brassus-Col du Marchairuz. 


\section{Résurgence de l'Orbe}

A $2 \mathrm{~km}$ au S-W de Vallorbe, la rivière sourd au pied d'une haute paroi de Malm, à l'altitude de $787 \mathrm{~m}$, dans le Séquanien. L'orifice s'enfonce obliquement sur une dizaine de $\mathrm{m}$, comme la branche ascendante d'un siphon.

La preuve a été faire que cette source est bien la résurgence des caux des lacs de Joux et Brenet qui disparaissent dans des «entonnoirs».

En 1776, une crue provoquée par la rupture d'un barrage troubla et enfla les eaux du lac Brenet; quelques heures plus tard, la source de l'Orbe augmenta son débit et se troubla.

En 1853/54, quelques mesures prouvent que les eaux des lacs et celles de la source présentent des variations correspondantes.

En 1884, l'ouverture des vannes de Bonport (lac Brenet) provoque une crue de l'Orbe au bout de 15 h. 30 .

En 1893, une coloration à la fluorescéine est complétée par une crue artificielle. Cette crue se manifeste déjà au bout de 2 h. 08 min., avec maximum 7 h. 40 min. plus tard. La coloration n'est apparue que $22 \mathrm{~h}$. plus tard et se maintint pendant $17 \mathrm{~h}$.

1894. Coloration des eaux à l'entonnoir du Rocheray (lac de Joux), avec effet sur la résurgence de l'Orbe au bout de 12 jours et pendant $24 \mathrm{~h}$.

1909. Le lac Brenet ayant désormais un écoulement artificiel, Forel constate que les températures varient encore dans des proportions sensiblement égales et en conclut que la résurgence est alimentée par les pertes des lacs dans la proportion de 30 à $40 \%$, le reste y étant amené par infiltration directe étendue aux quelque $300 \mathrm{~km}^{2} \mathrm{du}$ bassin supérieur de l'Orbe.

\section{Grottes-aux-Fées}

A $100 \mathrm{~m}$ au-dessus de la source s'ouvrent les Grottes-aux-Fées. La plus accessible est profonde d'environ $200 \mathrm{~m}$ et se termine par une cheminée conique de 60 à $70 \mathrm{~m}$ de hauteur. La seconde, plus profonde, est d'un accès plus malaisé. On peut penser qu'il s'agit là d'orifices antérieurs du cours souterrain de l'Orbe, mais leur direction peut laisser supposer leur rapport avec la vallée sèche du Crêt-Cantin.

\section{Col de la Pierre-Punex (1060 m)}

C'est depuis toujours la limite septentrionale de la vallée de Joux. C'est là que se rencontrent les territoires de Vallorbe (district d'Orbe) et ceux de deux communes du district de la Vallée: Le Lieu et l'Abbaye. La vallée de Joux a été occupée par un glacier local dont la présence a fait obstacle à toute glaciation d'origine alpine. Lorsque le glacier du Rhône commença à se retirer, le glacier de Joux put s'étendre quelque peu, débordant de sa cuvette par les points les plus bas, soit ici au col de la Pierre-Punex $(1060 \mathrm{~m})$, soit au col de Petra-Felix $(1150 \mathrm{~m})$, gagnant en étendue ce qu'il perdait en hauteur. Le glacier de Joux a dû finalement stationner longtemps à l'altitude de $1100 \mathrm{~m}$, franchissant encore le seuil de la Pierre-Punex et contribuant à creuser le petit bassin fermé du Mont d'Orzeires. La décrue continuant, le glacier resta enfermé dans la cuvette.

Les eaux de fusion du glacier devaient vraisemblablement disparaître par des orifices naturels résultant de l'érosion karstique antérieure. Ceux-ci ayant été colmatés, un lac se forma dont l'écoulement du trop-plein devait se faire par le seuil de la Pierre-Punex, à $1060 \mathrm{~m}$, jusqu'à l'ouverture des entonnoirs actuels qui ont ramené le lac au niveau de ces ouvertures.

Un bois de renne, trouvé dans un ancien delta de ce lac permet de préciser qu'il devait exister à la fin du Quaternaire.

\section{Entonnoirs du lac Brenet}

La vallée de Joux constitue un poljé, un bassin fermé dont les eaux s'écoulent par voie souterraine. Les «entonnoirs» les plus importants et les plus intéressants se situent sur la 


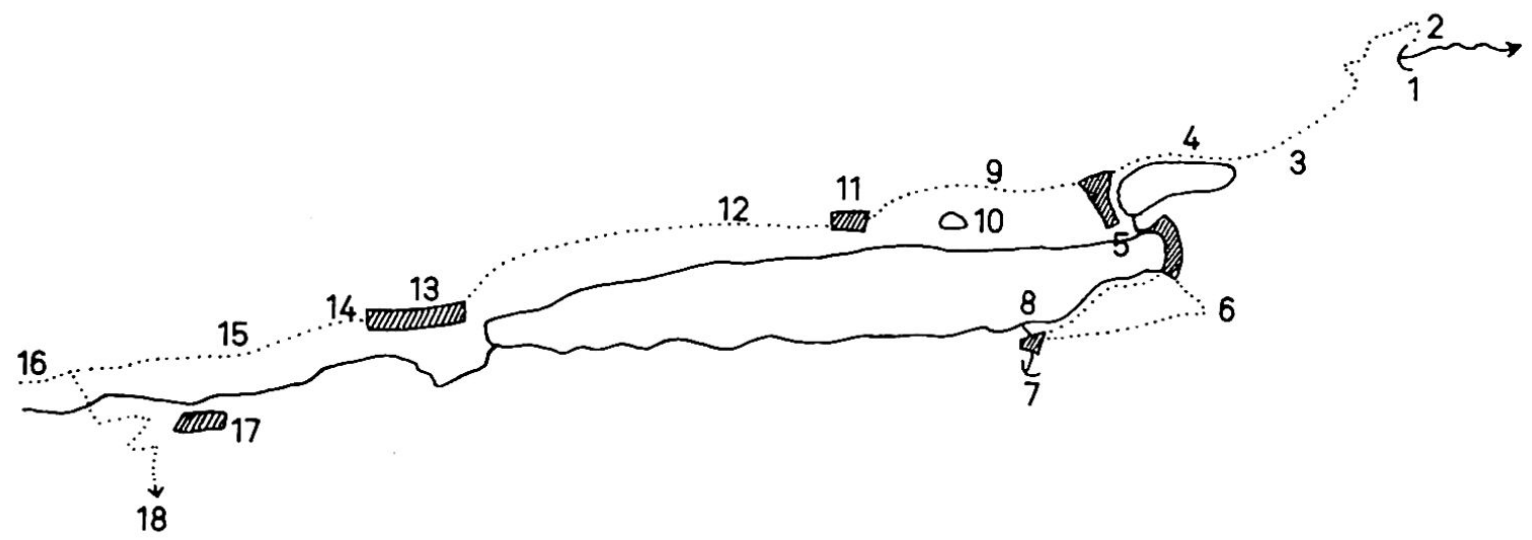

Itinéraire

rive des lacs. Le lac de Joux en compte une dizaine; ici au lac Brenet, il y en a quatre, en venant du N: la Cave à Metzi, Bon-Port, l'entonnoir Martinet, l'entonnoir Neuf. Le principal est celui de Bon-Port, au pied d'une paroi de Portlandien, à la limite du Purbeckien. Ces entonnoirs sont des puits plus ou moins verticaux, interrompus par des étranglements et des éboulis. Entre 1891 et 1893, des travaux ont été entrepris à BonPort pour élargir l'exutoire et faciliter l'écoulement des eaux. On a relevé alors l'existence de deux galeries, l'une de $20 \mathrm{~m}$, dirigée vers la montagne, l'autre, horizontale, aboutissant à une cheminée de $12 \mathrm{~m}$.

L'enfouissement des eaux du bassin supérieur de l'Orbe est un phénomène gros de conséquences. Si le réseau des eaux superficielles avait pu se maintenir, si l'Orbe avait suivi un cycle d'érosion normal, la vallée de Joux en eût perdu en partie ses deux caractères dominants: l'altitude et l'isolement. A remarquer que l'on n'a jamais vu les entonnoirs du lac Brenet refluer un trop-plein dans le lac, ce qui peut se produire, après de très fortes pluies coïncidant avec la fonte des neiges, pour les entonnoirs du lac de Joux.

Pour parer au manque d'eaux courantes, on eut très tôt recours à l'exutoire de BonPort pour en tirer une force motrice. Des installations industrielles (moulins, martinets) y furent établies déjà en 1524. En 1777 on construisit divers engins dans la cavité même de l'entonnoir et leur activité dura jusqu'en 1883, année où une crue souleva les constructions et les brisa dans l'abîme au retrait des eaux.

\section{Les Epinettes - entre les lacs}

D'ici la différence de niveau entre les deux lacs saute aux yeux. C'est une situation qui n'a rien de naturel. Jadis les deux lacs communiquaient librement par le cours de l'Orbe. On voit encore cet ancien lit, peu à peu comblé par les gadoues. En 1886, la voie ferrée étant construite du Day au Pont, il fallut créer les terrassements nécessaires à cette gare. (Le prolongement jusqu'au Brassus ne date que de 1899). A cette occasion un nouveau canal à niveau fut creusé sur le territoire du Lieu, alors que l'ancien fossé naturel délimite encore les deux communes du Lieu et de l'Abbaye.

Les travaux entrepris de 1901 à 1904 pour alimenter l'usine de Là-Dernier (Vallorbe) ont donné toute sécurité contre les risques d'inondation en cas de fermeture accidentelle des exutoires naturels. La galerie souterraine aboutissant au Crêt-des-Alouettes, d'où part la conduite forcée de l'usine, fixait un niveau minimum à $1005 \mathrm{~m}$ et un maximum à $1008,50 \mathrm{~m}$.

Des difficultés ont cependant surgi. En temps de sécheresse prolongée, les lacs cessaient de communiquer et il fallait avoir recours à l'installation coûteuse de pompes, 
aussi de nouvelles dispositions ont-elles été prises. Le lac Brenet est maintenu à un niveau de $1002 \mathrm{~m}$ et le canal entre les lacs étant muni de vannes, le lac de Joux sert de bassin de compensation (alt. max. $1005 \mathrm{~m}$ ). Une canalisation souterraine, avec dispositif de pompes, assure l'alimentation du lac Brenet. Il en est résulté pour le village des Charbonnières un appréciable gain de terrain utilisé en pâturage. Pour les riverains du grand lac, des conventions ont été signées par lesquelles ils sont assurés d'un niveau d'eau suffisant $(1 \mathrm{~m}$ de plus que le minimum fixé à $1001,50 \mathrm{~m}$ ) pendant les mois de mai à août qui sont les mois du tourisme d'été.

Dressé devant nous, le cheval ailé, l'énergie jaillissant des eaux, veut symboliser la fin de ces travaux.

Sous nos yeux, deux villages, Le Pont et Les Charbonnières. A remarquer que le district de la Vallée ne compte que trois communes: Le Chenit qui en occupe la partie supérieure, l'Abbaye et Le Lieu, séparées par les lacs. A l'origine il n'y avait qu'une seule commune, celle du Lieu qui apparaît constituée en 1396. En 1571 le territoire qui est aujourd'hui celui de l'Abbaye se détacha de la commune-mère. Son exemple fut suivi par les communiers du Chenit qui s'érigèrent à leur tour en commune en 1646. Mais à l'intérieur de chaque commune les villages ont conservé leur propre administration; ainsi L'Abbaye compte trois fractions de commune: L'Abbaye, Le Pont et Les Bioux. Le Lieu en compte aussi trois: Le Lieu, Le Séchey, Les Charbonnières, mais en a compté deux autres encore qui ont disparu avec le dépeuplement des hameaux isolés: Combenoire et la Fontaine-aux-Allemands. Le Chenit ne s'est subdivisé qu'au début de ce siècle et aussi en trois fractions: Le Sentier, L'Orient et Les Brassus. Ce fractionnement administratif tient à une cause géographique, l'isolement, la difficulté des communications pendant les longs et rudes hivers que connaît la région.

Quel est le caractère commun à ces deux villages qui épousent les rives de l'un et de l'autre lac? Ce sont des villages-routes, comme tous ceux que nous trouverons dans ce pays. Le Pont qui dérive son nom du moyen de communication établi très tôt sur le goulet des lacs alligne ses groupes de maisons, simples, doubles ou quadruples, d'un seul côté de la route, devenue un quai - promenade qui lui vaut, avec sa situation abritée et ensoleillée le renom de Montreux du Jura. Les groupes de maisons sont séparés par des ruelles rapides qui se continuent en dévestitures des champs. Une route nouvelle doublant celle du bord du lac fait surgir des constructions qui n'ont plus aucun rapport avec l'architecture traditionnelle. Aux Charbonnières, dont l'origine remonte au moulin bâti sur le ruisseau de la Sagne, c'est la bifurcation de la route vers Mouthe et vers le Lieu qui a ordonné le village. Tout ce qui borde le lac, terriblement exposé à la bise, est de construction récente (avec les anciens moyens de chauffage on était plus prudent); ainsi l'agglomération a rejoint le petit voisinage des Crettets, sous la gare. Le village a été incendié en partie en 1866, en 1872 et encore en 1900, aussi ne compte-t-il plus guère de maisons anciennes. Quant à son nom, il rappelle l'exploitation des forêts par les charbonniers pourvoyeurs de l'industrie métallurgique de Vallorbe. L'horlogerie n'a pas pris dans ces deux villages le développement qu'elle connaît au Chenit, mais elle y est cependant représentée par quelques entreprises. Le village des Charbonnières a été le premier centre suisse de la fabrication des fromages en boîte, à pâte molle, dits vacherins. Le Pont, nœud routier, a misé sur le tourisme.

\section{Le Mont-du-Lac $(1108 \mathrm{~m})$}

Ce point de vue d'où l'on prend en enfilade tout le bassin supérieur de l'Orbe nous permet de comprendre la structure de la région.

Les grandes lignes du relief sont données par la structure géologique. D'une manière générale les chaînes correspondent aux anticlinaux et les vallons aux synclinaux. A l'Est se dresse l'anticlinal du Mont-Tendre $(1679 \mathrm{~m})$ qui porte le plus haut sommet du Jura suisse. Ce pli qui domine la plaine vaudoise est large de $8 \mathrm{~km}$. 


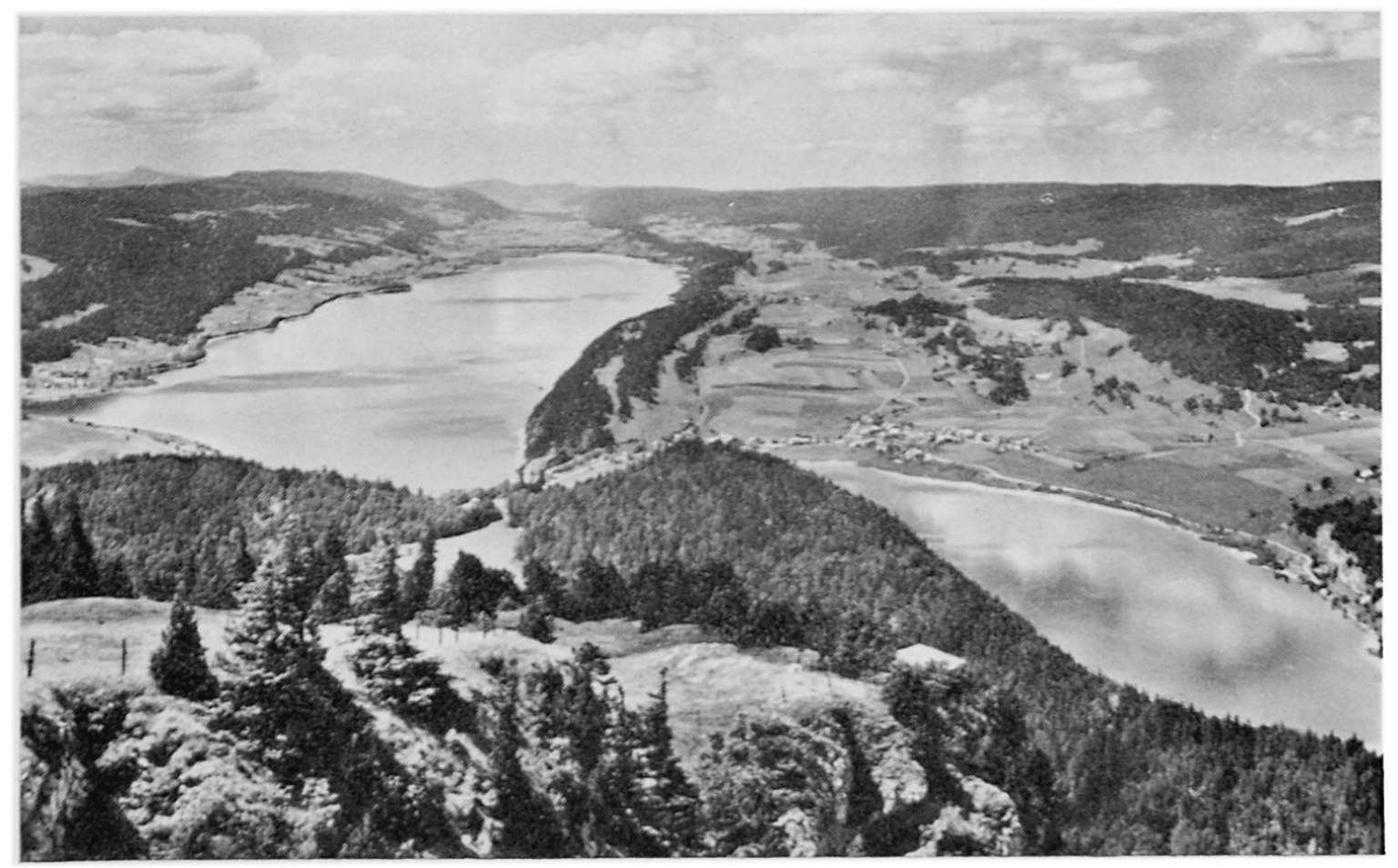

Vallée de Joux. Les Lacs de Joux (en arrière) et Brenet vue du Sommet de la Dent de Vaulion.

Photo: M. Deriaz, Vallorbe

Devant nous, le lac de Joux, profond de $32 \mathrm{~m}$, occupe le synclinal principal; en amont du lac, la plaine alluviale où sinue l'Orbe. La rive uccidentale du lac de Joux est formée par un anticlinal étroit qui sépare le sýnclinal de Joux d'un synclinal occupé en son point le plus bas par le lac Brenet; enfin, fermant l'horizon à l'Ouest, le large dôme anticlinal du Risoud.

Ainsi devant nous la belle ordonnance des plis... derrière nous, l'accident qui a bouleversé ce bel ensemble.

En effet, toute la région a été disloquée par le grand décrochement transversal qui se manifeste de Montricher à Vallorbe et Pontarlier. L'anticlinal Haut-de-Molendruz Dent-de-Vaulion, issu de la chaine du Mont-Tendre s'est déversé en chevauchant le synclinal de Joux.

Les collines qui ferment le bassin, l'Aouille $(11+7 \mathrm{~m}$ ) et les Agouillons (1162 et $1217 \mathrm{~m}$ ) sont constituées par les masses jurassiques renversées du flanc occidental du pli de la Dent-de-Vaulion.

\section{Source de la Lyonne}

C'est une source vauclusienne jaillissant d'une paroi de calcaire portlandien. Deux exutoires anciens, un peu plus haut, ne fonctionnent qu'en cas de pluies violentes. Alors le débit de la Lyonne augmente considérablement au bout de quelques heures. Rien ne permet de déceler l'origine de ces eaux $(6,2)$. Bien que son cours soit très bref $(600 \mathrm{~m})$, la Lyonne rend de grands services. Des scieries sont établies dans la cluse qu'elle a ouverte dans le Crétacé. En 1480 Vinet Rochat obtint de l'abbé de Joux la concession de la Lyonne pour y établir martinets, forges et hauts fourneaux. Une prise d'eau et un bief lui permettent encore de contribuer à l'activité de la fabrique de limes de l'Abbaye.

\section{S. L'Abbaye}

C'est sur le delta formé par la Lyonne que fut édifié le monastère fondé en 1162 , suivant la règle de Prémontré. Appelé Lcona, puis Domus Dei, abbaye de Cuarnens, 
enfin abbaye du lac de Joux, le monastère, supprimé en 1536, n'a laissé qu'une tour, antérieure au XIIIe siècle, seul vestige du moyen âge à la Vallée.

En revenant au Pont par le bord du lac, on peut observer un amas fluvio-glaciaire avec beine qui pourrait être le témoignage d'un stade de reirait du lac de $1060 \mathrm{~m}$.

\section{Le Séchey}

En quittant les Charbonnières on constate bientôt que le synclinal du Lieu a un relief très irrégulier: collines allongées voisines de dépressions humides; ces formations crétacées et tertiaires sont recouvertes généralement des dépôts morainiques qui leur valent de porter de belles prairies.

Le village, en contre-bas de la colline qui le protège de la bise, montre encore de ces alignements de maisons mitoyennes que les incendies ont fait disparaitre si souvent, mais qui étaient jadis le dispositif normal. La maison unitaire tripartite est caractérisée à la Vallée par le «néveau», espace ouvert ou fermé qui permet de communiquer, à l'abri, du logis à la grange et à l'écurie.

\section{Le lac Ter}

Anciennement Laytet (petit lac), il occupe le fond d'une cuvette colmatée par les matériaux morainiques. C'est le seul de ce genre qui subsiste dans la région avec le lac des Rousses; partout ailleurs la végétation a envahi ces petites nappes d'eau et les a transformées en tourbières.

\section{Le Lieu}

Le Lieu est le plus ancien village de la Vallée. Il doit son origine et son nom à Pontius ou St-Point, moine de Condat (St-Claude). Le Lieu (Locus Domini Pontii) est qualifié de village en 1324 , compte 30 chefs de famille en 1386 et la communauté apparaît organisée dix ans plus tard. Le village fut presque entièrement détruit par le feu en 1691, puis il perdit 34 maisons en 1858 et 12 en 1882 . L'agriculture, l'élevage, l'industrie du bois et l'horlogerie se partagent l'activité des habitants.

\section{Combenoire}

Les maisons isolées, autrefois nombreuses sur le territoire du Lieu, ont en grande partie disparu. La concentration de la population au détriment des écarts est un phénomène que les statistiques ne mettent pas en évidence. Il apparaît ici à Combenoire mieux que partout ailleurs.

\section{Le Sentier}

La route quitte la combe du Lieu pour passer dans la vallée principale par la petite cluse de la Golisse. Aussitôt c'est le monde de l'industrie qui apparaît. De la Golisse à Chez-le-Maitre et aux Piguet-Dessous, en effet, s'allonge la plus considérable des agglomérations de population qui soit à la Vallée. De part et d'autre de ce qui fut le «sentier», origine du nom de la localité, et qui est aujourd'hui une grande artère de circulation, s'égrènent des constructions modernes qui ont remplacé les maisons rurales des premiers exploitants du sol. La vie industrielle se manifeste par les grandes manufactures aux façades vitrées, les maisons ouvrières du type locatif et les cossues maisons familiales. L'habitat, d'abord limité au pied de la Côte, a pris d'assaut le flanc de la montagne et les routes qui traversent la plaine de l'Orbe vers l'Orient et le Brassus sont à leur tour des axes de peuplement dont l'importance va encore augmenter à la suite des travaux de régularisation de la rivière.

L'église bâtie dès 1898 remplace le bâtiment incendié que les communiers avaient édifié à grand peine en 1612. L'hôtel-de-ville battant neuf est sur l'emplacement de l'ancien.

\section{Chez-le-Maître}

Ce hameau devient de plus en plus le centre de la commune du Chenit (origine: tseneu $=$ branche de foyard $\mathrm{sec}$ ). C'est là que se trouvent réunis l'hôpital, le Collège et 
FIG. 21 Anticlinal du Risoud

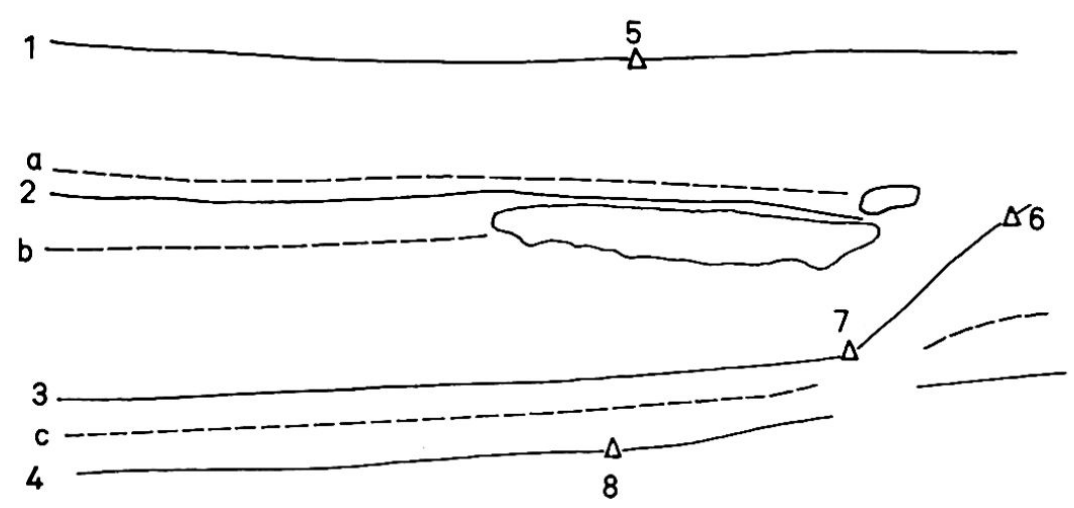

2 Anticlinal de la Côte

3 Anticlinal du Haut-deMolendruz-Dt. de Vaulion

4 Anticlinal du Mt. Tendre

5 Grand Crèt $(1419 \mathrm{~m})$

6 Dt. de Vaulion (1483 m)

7 Haut-de-Molendruz (1439 m)

8 Mt. Tendre $(1679 \mathrm{~m})$

a) Synclinal du Solliat-Brenet. b) Synclinal de Joux. c) Synclinal des Crosets-Vaulion

l'Ecole d'horlogerie et de mécanique, cette dernière assurant la formation d'une maind'œuvre qualifiée. L'Ecole possède un musée riche de pièces remarquables dont la plupart ont été créées par des artisans de la région. L'industrie horlogère a pris pied à la Vallée au milieu du XVIIIe siècle. Cette industrie avait été précédée par la fabrication d'horloges de bois ou de métal, à l'imitation de ce qui se faisait à Morbier et Bellefontaine. En 1740, Samuel-Olivier Meylan se rendit à Rolle et y apprit en trois ans le métier d'horloger. Revenu au pays, il forma des apprentis et le métier ne tarda pas à se répandre dans toute la Vallée. Les horlogers fabriquaient alors la montre complète et ceux de la vallée de Joux ne tardèrent pas à se distinguer, surtout sur la place de Genève. Ces artisans-paysans consacraient leurs longs loisirs forcés à la confection des pièces les plus compliquées. A la fin du siècle on en vint à la division du travail. La fabrication mécanique aurait pu porter un coup mortel à une industrie qui avait exigé avant tout l'habileté manuelle. C'est alors que fut fondée en 1833, au Sentier, la fabrique Lecoultre qui est demeurée la plus importante et occupe plus de 800 personnes. Les manufactures n'ont cependant pas fait disparaître entièrement le travail à domicile.

\section{Chez-Tribillet}

A la sortie des Piguet-Dessous on retrouve une petite cluse à travers la côte, semblable à celle de la Golisse, mais qui possède un ancien delta formé dans le lac de $1060 \mathrm{~m}$ par un torrent nourri par les glaciers et névés du Risoud. Il est exploité en gravière et c'est là qu'a été retrouvé le bois de renne prouvant l'existence de ce lac à la fin du quaternaire.

L'absence de graviers lacustres en amont permet de croire que le glacier existait encore dans la partie supérieure de la vallée alors que le lac en occupait la partie inférieure.

A trois $\mathrm{km}$ de là cesse la zone agricole. Tout le fond de la vallée de Joux, jusqu'à la frontière, n'est que bois ou pâturage. Ce n'est pas une limite d'altitude puisque l'agriculture reprend ses droits au delà de la frontière; c'est une limite historique. La colonisation par les gens de la Vallée a été stoppée par l'acquisition de ces terres utilisées comme pâtures d'été par des communes du pied du Jura, Bursins et Burtigny, d'où ces pâturages tirent leur nom.

De plus, la politique de défense militaire des frontières telle que la concevait Berne y a maintenu une zone boisée.

\section{Pra-Rodet}

Le retrait du glacier de Joux a laissé dans la partie amont de la vallée des éléments de moraines, soit collines allongées dans l'axe de direction, restes de moraines latérales, sur lesquelles l'habitant s'est volontiers établi, soit arcs de moraines frontales qui ont 
facilité la construction de routes transversales grâce à leur surélévation par rapport au fond de la vallée. L'exemple le mieux conservé est celui de Pra-Rodet qui enserre une tourbière boisée.

\section{Le Brassus}

Traversons l'Orbe pour gagner le Brassus où s'amorce la route du Marchairuz.

Le village tire son nom de la petite rivière dont la source, abîmée par les travaux de captage, n'a cessé de fournir à la localité l'énergie pour ses industries et l'eau potable à une bonne partie de la commune du Chenit.

Le premier établissement industriel date de 1555. Pendant longtemps, les usiniers ont utilisé le minerai de fer du Bas-du-Chenit, du Risoud et même celui des Charbonnières qu'on amenait par des radeaux. Les usines métallurgiques ont travaillé jusqu'en 1827. Elles ont été remplacées par des scieries. L'horlogerie et ses industries annexes font la prospérité du village. Enfin le Brassus est devenu un centre sportif. Le tremplin de saut de la Chirurgienne, le plus grand de Suisse, voit accourir chaque année les skieurs des disciplines nordiques à l'occasion des grands concours internationaux qui ont lieu chaque année depuis 1955.

Avant de quitter la Vallée, il convient de jeter un coup-d'œil sur le large pli boisé qui ferme l'horizon à l'ouest. La forêt du Risoud s'étale sur une longueur de $20 \mathrm{~km}$ et a une largeur variable, mais s'étendant jusqu'à $3 \frac{11}{2} \mathrm{~km}$ au sud-ouest. Avec ses 2386 ha, c'est la plus vaste forêt suisse d'un seul tenant. Elle a toujours été considérée comme un élément important de la défense militaire du pays, un de ces «bois d'avenue» destinés à couvrir la frontière du côté de la Franche-Comté. Elle devint propriété de l'Etat de Vaud en 1803, mais les habitants y avaient droit de bocherage depuis 1186; alors pour mettre fin à une exploitation préjudiciable à la forêt, l'Etat de Vaud accorda en toute propriété aux trois communes 854 ha que celles-ci se partagèrent en 1910. Les épicéas constituent $80 \%$ du peuplement, le reste étant composé de sapins blancs et de hêtres. La pauvreté du sol et la rigueur du climat (montée de la sève en juin, retrait de la sève en septembre) font que la croissance des arbres est ralentie, réduite à moins de $1 \mathrm{~mm}$ par année. Ces conditions font des bois d'une texture très fine et d'une dureté exceptionnelle, très recherchés par diverses industries.

\section{Col du Marchairuz (1447 m)}

Au XIIIe siècle les relations de la Vallée avec le Pays-de-Vaud se faisaient surtout par le Molendruz. L'horlogerie ayant créé de fréquentes relations avec Genève, l'ouverture d'une route carrossable par le Marchairuz s'est imposée. Ce fut fait en 1769. Le trafic est resté assez intense jusqu'à la construction de la voie ferrée de Lausanne à Cossonay qui a rendu au Molendruz son importance et qui reste ouvert au trafic, hiver comme été, grâce à sa moindre altitude $(1180 \mathrm{~m})$.

\section{RESUME}

Le Jura est coupé par plusieurs décrochements transversaux. Entre deux de ces décrochements, celui de St-Cergue-Morez et celui de Montricher-Vallorbe-Pontarlier, les plis jurassiens s'ordonnent régulièrement, les chaînes correspondant aux anticlinaux et les vallées aux synclinaux. Le trait le plus original de la vallée de Joux est d'être un bassin fermé, le plus grand qui soit dans le Jura suisse avec ses $170 \mathrm{~km}^{2}$ (non compris sa partie française). Ce bassin fermé résulte de la dislocation de la Dent-de-Vaulion qui a obstrué la synclinal de Joux et aussi de l'érosion karstique; l'écoulement souterrain des eaux a maintenu le bassin à haute altitude. Le colmatage glaciaire est venu troubler cette érosion et a permis la formation des lacs. L'altitude et la nature de bassin fermé déterminent un climat rude, aux hivers rigoureux.

La nature du terrain est généralement trahie par la végétation; la forêt occupe les calcaires et les éboulis rocheux, alors que les moraines ou les terrains alluviaux ont été utilisés par l'homme, aménagés surtout en prés et pâturages.

La colonisation n'est intervenue qu'au moyen âge, par la fondation d'établissements monastiques. La population est encore clairsemée au XVIe siècle. L'ouverture des routes vers la 
«Plaine», au XVIIIe siècle et l'introduction de l'industrie horlogère à la même époque vont modifier les conditions de vie, concentrer la population, transformer les habitations, créer ce type de l'artisan-paysan qui vient de disparaître par la mécanisation du travail en fabrique. L'horlogerie qui a éclipsé toutes les autres activités est aujourd'hui l'industrie qui fait vivre la majeure partie des 7500 «Combiers».

\section{STATISTIQUES}

\section{Climat}

Moyenne annuelle: $5^{\circ}$

Moyenne des 3 mois d'été : $12^{\circ} 9$

Moyenne des 3 mois d'hiver: $-3^{\circ}$

Mois le plus chaud (juillet) : moyenne $14^{\circ}$

Mois le plus froid (janvier) : moyenne $4^{\circ}$

Amplitude des moyennes: $18^{\circ}$

Amplitude annuelle des extrêmes: $50^{\circ}$

Amplitude des extrêmes observés: $72,7^{\circ}$ (-41 le 31.1 .88 et $+31,7^{\circ}$ le 19.8.98)

Congélation des lacs, de fin décembre au début d'avril.

Enneigement de novembre à mars-avril.

Précipitations: $2 \mathrm{~m}$ sur le Risoud; $1,5 \mathrm{~m}$ au fond de la vallée.

Prédominance générale des vents du S-W et, en hiver, des vents du N-E.

\section{Hydrographie}

Lac de Joux, alt. $1004 \mathrm{~m}$; superf. $8,6 \mathrm{~km}$ prof. $32 \mathrm{~m}$.

Lac Brenet, alt. $1002 \mathrm{~m}$; superf. o, $6 \mathrm{~km}$; prof. $18 \mathrm{~m}$.

Cours suisse de l'Orbe: $10 \mathrm{~km}$; pente $3,2 \%$, débit $3 \mathrm{~m}^{3} / \mathrm{sec}$.

\section{Superficie}

15906 ha, dont 1373 en prés, 802 en champs, 5484 en forêts et 8200 en pâturages.

Population en 1961

$\begin{array}{lc}\begin{array}{l}\text { Commune de l'Abbaye: } \\ \text { dont }\end{array} & 1110 \text { Suisses } 86 \text { Etrangers }=1196 \mathrm{~h} . \\ \text { L'Abbaye } & 21 \text { o Suisses } 17 \text { Etrangers }=227 \mathrm{~h} . \\ \text { Les Bioux } & 551 \text { Suisses } 45 \text { Etrangers }=596 \mathrm{~h} \text {. } \\ \text { Le Pont } & 349 \text { Suisses 24 Etrangers }=373 \mathrm{~h} . \\ \text { Commune du Lieu: } & 888 \text { Suisses } 75 \text { Etrangers }=963 \mathrm{~h} . \\ \text { dont } & 399 \text { Suisses } 33 \text { Etrangers }=432 \mathrm{~h} . \\ \text { Le Lieu } & 372 \text { Suisses 38 Etrangers }=410 \mathrm{~h} \text {. } \\ \text { Les Charbonnières } & 117 \text { Suisses 4 Etrangers }=121 \mathrm{~h} \text {. } \\ \text { Le Séchey } & \\ \text { Commune du Chenit: (1963) } & \end{array}$

Le Sentier: 2867

L'Orient : 900

Le Brassus: 1686

dont 514 Italiens, 228 Espagnols, 145 Français.

Mouvement de la Population

$\begin{array}{ccccc} & \text { 'L'Abbaye } & \text { Le Chenit } & \text { Le Lieu } & \text { La Vallée } \\ 1850 & 1018 & 2766 & 999 & 4783 \\ 187 \text { o } & 1204 & 3619 & 1108 & 5931 \\ 1888 & 1064 & 3474 & 989 & 5527 \\ 1910 & 1157 & 4118 & 1037 & 6312 \\ 1930 & 1149 & 4018 & 917 & 6084 \\ 1950 & 1142 & 4604 & 1020 & 6766 \\ 1960 & 1142 & 5328 & 979 & 7449\end{array}$




\begin{tabular}{|c|c|c|c|c|}
\hline & 营 & 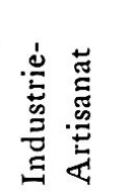 & 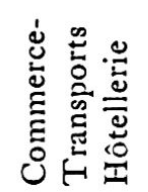 & 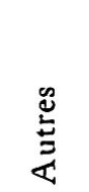 \\
\hline L'Abbaye & 53 & 337 & 53 & $\begin{array}{r}51 \\
260\end{array}$ \\
\hline $\begin{array}{l}\text { Le Chenit } \\
\text { Le Lieu }\end{array}$ & $\begin{array}{r}141 \\
74\end{array}$ & $\begin{array}{r}1584 \\
294\end{array}$ & $\begin{array}{r}192 \\
55\end{array}$ & $\begin{array}{r}260 \\
60\end{array}$ \\
\hline La Vallée & 268 & 2215 & 300 & 371 \\
\hline
\end{tabular}

\title{
DER STRUKTURWANDEL DER ALPWIRTSCHAFT IM LUFTBILD (AM BEISPIEL DES DISCHMATALES)
}

\author{
HAROLD HAEFNER
}

\section{DAS PROBLEM}

Die Strukturwandlungen der Alpwirtschaft und die Veränderungen im alpinen Landschaftsbild in jüngster Zeit sind bekannt und mehrfach beschrieben worden ( $\mathrm{Pa}$ schinger 1962, Suter 1963, Gutersohn 1963, 1964 usw.). Es handelt sich vorwiegend um Extensivierungsvorgänge, um Bevölkerungsabwanderungen, Änderungen in der Wirtschaftsform (Aufgabe der jahreszeitlichen Wanderungen, Aufgabe der höchst- und entferntest gelegenen Alpen, Wechsel von Kuh- in Jungvieh- oder Schafalpen usw.). Andererseits wird versucht, durch tiefgreifende Sanierungen geeigneter Objekte rationellere Nutzung und Qualitätsverbesserung zu erreichen (Melioration, Wegerschließung, Bau von Transportbahnen und Milchpipelines, Stallsanierung, Weideverbesserung, Erstellung von Verschlauchungsanlagen usw.). Die Extensivierungsvorgänge sind bedeutend und nehmen in den letzten Jahren an Umfang eher noch zu. Die positiven Strukturänderungen dagegen sind viel geringer und werden nur zögernd und in unzulänglicher Weise an die Hand genommen.

Der allgemeine Trend ist bekannt. Über das eigentliche Ausmaß der Vorgänge aber macht man sich kaum eine Vorstellung, da bisher nie versucht wurde, diese Phänomene in ihrer räumlichen Verbreitung und zeitlichen Abfolge exakt festzuhalten. Gerade derartige Untersuchungen sind aber notwendig, um uns ein klares Bild über die Bedeutung dieser Vorgänge und die Dringlichkeit zu energischer Hilfe vor Augen zu führen. Die kartographische Erfassung dieser Strukturänderungen verursacht auch bedeutende Schwierigkeiten. Hier kann nun das Luftbild sinnvoll eingesetzt werden und außerordentlich wertvolle Dienste leisten. Oft wird es sogar die einzige Quelle sein, die uns genauere Angaben über diese Veränderungen in der Wirtschaftslandschaft zu geben vermag. Die sich hier bietenden Möglichkeiten und die anzuwendenden Arbeitsmethoden sollen im folgenden für die Belange der Alpwirtschaft besprochen und anhand eines praktischen kleinen Beispiels erläutert werden.

\section{DIE MÖGLICHKEITEN DER LUFTBILDINTERPRETATION}

Im Luftbild besitzt die geographische Forschung ein wertvolles kulturhistorisches Dokument, das einen einmaligen und nicht wiederholbaren Zustand der Landschaft zu 